\title{
Vocación docente e influencia en el aprendizaje en escolares en tiempo de pandemia
}

\author{
Johanna Raquel Aguilar Insfrán \\ joha7112013@gmail.com \\ Facultad de Humanidades y Ciencias de la Educación, \\ Universidad Nacional de Pilar.
}

\section{RESUMEN}

La investigación tiene como objetico determinar la vocación docente, su influencia en el aprendizaje en tiempo de pandemia de niños/as de la Escuela Básica $N^{o} 578$ "Defensores del Chaco", San Lorenzo - Pilar, año 2020-2021. En relación al tipo de investigación, se aplicó la investigación descriptiva, con enfoque cualitativo y cuantitativo. La muestra es de 231 alumnos de la Educación Escolar, 22 docentes, 1 evaluadora 1 director, a través de los métodos cualitativo y cuantitativo. Las técnicas de recolección de datos que se implementó el cuestionario dicotómico. Llegando a la conclusión de la gran influencia que tiene la vocación docente en el aprendizaje de los alumnos, en la que interviene la aptitud, la voluntad del docente, el desempeño que pueda poner en el aprendizaje de los alumnos, dando seguimiento a los alumnos y evitar la deserción escolar, en colaboración con directivos, colegas y padres de familia, como así también en caso necesario solicitar la intervención de organismos del estado. De esta manera se ha llegado a confirmar la hipótesis que plantea: La vocación docente, el acompañamiento coordinado de padres o tutores con los docentes y organismos del estado influyen de manera positiva en el aprendizaje de niños/as de la Escuela Básica $\mathrm{N}^{\circ}$ 578 "Defensores del Chaco", San Lorenzo Pilar, año 2020-2021, en tiempo de pandemia.

Palabras claves: vocación docente; desempeño docente; enseñanza-aprendizaje; aprendizaje en tiempo de pandemia. 


\title{
Teaching vocation and influence on learning in schools in time of pandemic
}

\begin{abstract}
The objective of the research is to determine the teaching vocation and, its influence on learning in times of pandemic of children from Basic School No. 578 "Defensores del Chaco", San Lorenzo - Pilar, year 2020-2021. In relation to the type of research, descriptive research was applied, with a qualitative and quantitative approach. The sample is 231 students of School Education, 22 teachers, 1 evaluator 1 director, through qualitative and quantitative methods. The data collection techniques that the dichotomous questionnaire was implemented. Coming to the conclusion of the great influence that the teaching vocation has on the students 'learning, in which the aptitude, the will of the teacher intervenes, the performance that can be put in the students' learning, monitoring the students and avoid school dropout, in collaboration with managers, colleagues and parents, as well as, if necessary, request the intervention of state agencies. In this way, the hypothesis that it raises has been confirmed: The teaching vocation, the coordinated accompaniment of parents or tutors with teachers and state agencies have a positive influence on the learning of children of Basic School No. 578 "Defensores del Chaco", San Lorenzo Pilar, year 2020-2021, in times of pandemic.
\end{abstract}

Keywords: teaching vocation; teaching performance; teaching-learning; learning in times of pandemic.

Artículo recibido: 15 octubre. 2021 Aceptado para publicación: 18 noviembre 2021 Correspondencia: joha7112013@gmail.com Conflictos de Interés: Ninguna que declarar 


\section{INTRODUCCIÓN}

Según Larrosa Martínez, (2010). Tradicionalmente la vocación ha estado indisolublemente unida al maestro y el enseñar se ha considerado un arte. Estas dos ideas, que gozaron de unanimidad durante mucho tiempo y que permanecen todavía en la mente de muchas personas, han evolucionado obligatoriamente con los cambios sociales. En el estudio de la vocación docente versus profesión se entremezclan dialécticamente elementos que han influido y están influyendo en el quehacer profesional del profesorado: la tradición, las transformaciones sociales, los avances de la investigación y las necesidades del profesorado.

Son muchos los que subrayan la importancia de la vocación en cualquier actividad profesional, tanto por razones de un mayor rendimiento en el trabajo como para evitar fracasos personales en el desempeño. Creen que la vocación determina las personas más adecuadas para una profesión y que existe la profesión más conveniente para cada persona.

Del mismo modo, para acceder a una profesión se deben poseer unas cualidades o aptitudes previas y en la elección de un trabajo hay que tener en cuenta la vocación. Sólo se es un verdadero profesional cuando se tiene vocación (Gichure, 1995, p. 210, mencionado por Larrosa Martínez, 2010).

En el caso de la enseñanza es evidente que se necesitan determinadas competencias para desempeñarla con corrección y que no todas las personas están capacitadas para ejercer estas funciones (García Garrido, 1999: p.436) resalta que "no todo el mundo sirve para esta profesión, en contra de lo que tan a menudo se cree; hace falta tener el perfil personal adecuado". Aunque, ciertamente, tendríamos que añadir que el perfil docente demandado está al alcance de todos los que deseen dedicarse al magisterio con voluntad, conocimiento y desempeño ético.

La vocación influye en el rendimiento escolar como también en la retención escolar, siendo que los docentes pueden influir en gran manera no solo en el aprendizaje sino también evitar el fracaso escolar, por lo tanto, en la retención del alumno en este tiempo de pandemia; por lo que la investigación estudia la vocación docente y su influencia en el aprendizaje de niños/as de la Escuela Básica No 578 "Defensores del Chaco", San Lorenzo - Pilar, año 2020-2021, en tiempo de pandemia. 
La vocación tiene dimensiones tales como las características de la personalidad eficaz, como son la autoestima, asertividad, capacidad de trabajo, confianza en sí mismo, estabilidad emocional, estilo de pensamiento, extraversión, flexibilidad, independencia, iniciativa, locus de control, motivación de logro, optimismo, perseverancia, tolerancia a la incertidumbre, toma de riesgos y valores personales (Martín, Martín, Dapelo, Pizarro, Gusto \& otros, 2015).

La vocación está estrechamente relacionada con la motivación, está última se fomenta desde las primeras etapas de la vida. En la motivación confluyen factores personales, psicológicos y sociales como: las experiencias familiares, el entorno en que se desenvuelve el individuo y la formación educativa (Fernández, González, Pompa Garcés, Figueredo, 2016); (Pérez, Rodríguez, 2016).

En la actualidad, cada vez se hace más difícil la retención del alumno en el sistema educativo, sobre todo en los sectores más vulnerables como son las instituciones educativas de las periferias de la ciudad, donde acuden alumnos con posibilidades económicas y sociales menos favorecidas y en la que se requieren docentes con vocación y el acampamiento de padres o tutores como también de organismos del estado como la Consejería de la Niñez y la Adolescencia y en caso necesario de la Fiscalía para que con un trabajo mancomunada se pueda lograr la retención y el éxito académico de los alumnos.

\section{REVISIÓN BIBLIOGRÁFICA}

\section{La orientación vocacional}

La orientación profesional constituye una actividad vital en la preparación de los jóvenes de cara a su inserción laboral, lo afirma (Ávila, 2016). La orientación vocacional y profesional es una actividad esencial del proceso educativo, que interesándose por el desarrollo integral del alumno, individual y socialmente considerado, le ayuda en la mejora de su conocimiento y dirección personal, para lograr su desarrollo equilibrado y para que con sus características propias tome su decisión vocacional, defina su carrera profesional, se comprometa a luchar para tener un mejor futuro y participe de una manera eficaz en la vida comunitaria (Velásquez, Puentes, Sarabia, 2014).

En concordancia con lo expuesto (Álvarez, 1995), al referirse a la Orientación Vocacional también como un proceso de desarrollo de la carrera a lo largo de la vida de 
las personas afirma a que está presente en los diferentes contextos en que se encuentren éstas, a saber

- El Educativo: que abarca el período formativo, es decir la preparación para la vida laboral y que se desarrolla fundamentalmente en el contexto escolar y comunal.

- Dentro de este contexto la Orientación Vocacional desarrolla diferentes acciones con el estudiantado, padres, madres, personas encargadas y docentes con el fin de favorecer el desarrollo integral del estudiantado y dentro de éste el vocacional.

- El Organizacional: incluye el período de desempeño laboral que tiene lugar en el centro de trabajo y en el cual se dan gran parte de las experiencias de las personas relacionadas precisamente con el trabajo, el cual cumple una doble función, tanto a nivel individual como social, de ahí su importancia.

Uno de los principales problemas que se plantean los estudiantes de nivel secundario al concluir sus estudios consiste en escoger correctamente la profesión a seguir (Medina, Watanabe, Angulo, 2018). La orientación vocacional surge por la necesidad de facilitar una información que oriente al individuo para lograr las competencias que requiere para insertarse en un contexto social laboral (González, Cardentey, 2015).

\section{La vocación}

Existen varios factores que contribuyen a caracterizan la presencia de la vocación del ser humano en la profesión que de desempeña, uno de los factores de relevancia es el conjunto de atributos de la personalidad en el desarrollo académico, vocacional y social de los estudiantes (Martín, Martín, Dapelo, Pizarro, Gusto \& otros, 2015). También la autoestima, la pasión con que se realiza el trabajo y la calidad del servicio prestado es determinante en el desarrollo profesional, la visión que tendrá el resto del equipo de salud y la sociedad. Por tanto, no habrá mejor demostración de valía que la competencia y desempeño (Fernández, González, Pompa Garcés, Figueredo, 2016).

La motivación, es también uno de los factores que determinan la profesión del estudiante y ayuda a conocer la vocación para la misma, se trata de un conjunto de procesos implicados en la activación, dirección y persistencia de la conducta. Algunos autores asumen que la motivación es la compleja integración de procesos psíquicos que efectúa la regulación inductora del comportamiento, pues determina la dirección (hacia el objeto-meta buscado o el objeto evitado), la intensidad y el sentido (de aproximaciónevitación) del comportamiento (Pérez, Rodríguez, 2016). 


\subsection{La educación y vocación}

Mendoza, Machado, Montes, (2016), plantean que la educación debe abordarse en el conocimiento considerando el contexto, lo global, lo multidimensional y lo complejo. Estos principios son pertinentes a la elaboración de los proyectos personales de vida y la elección de la profesión.

El conocimiento de sí mismo y la elección vocacional se van estructurando mutuamente.

El concepto de sí mismo se desarrolla durante la vida del sujeto a través de fases y, una vez reconocida internamente, el sujeto opta por escoger lo que más le gusta hacer. En el presente estudio, este factor es el que ha dado resultados más ajustados con la realidad, los que son reflejados en la relación entre autoconfianza y rendimiento académico (Medina, Watanabe, Angulo, 2018).

La adecuada formación vocacional, orientación profesional y reafirmación de la vocación en los niños, adolescentes y jóvenes, asegura en gran medida el ingreso con calidad tanto al centro de formación profesional como laboral (González, Cardentey, 2015).

\subsubsection{Vocación y condiciones de trabajo}

Las condiciones laborales de los docentes es un tema muy escasamente abordado por la comunidad educativa, porque siempre se ha considerado que el docente se conforma con lo mínimo que el Estado ofrece para mejorar las condiciones educativas, esto no solo en Paraguay, pues es similar en los otros países latinoamericanos, tales como Chile, Argentina, Perú y otros que a continuación se muestran algunos resultados.

En relación a las jornadas laborales que desarrollan los docentes fuera del horario pagado, manifestaron, según una encuesta realizada en Chile: "las principales tareas que se realizan fuera del horario de trabajo son: preparación de material didáctico $(87.1 \%)$; cursos de perfeccionamiento $(85.5 \%)$; preparación de clases $(83.2 \%)$.

Frente a esta carga de trabajo y los tiempos destinados a ella, destaca como factor de riesgo para la salud la ausencia de tiempos de descanso adecuados: casi un $45 \%$ de los profesores destina menos de 15 minutos al descanso durante la jornada laboral. Asumiendo como normal una jornada de trabajo de 44horas semanales, el tiempo libre disponible para los profesores (sumados el trabajo docente realizado en la escuela y en el hogar) debería ser de 124 horas, las que serían de libre disposición para dormir, 
realizar actividades distintas a la docencia, trabajo doméstico, vida familiar. En esa condición ideal teórica se encontraría el 52\% de los profesores. Sin embargo, al considerar en el cálculo de horas de tiempo libre las horas destinadas a trabajo doméstico y a desplazamiento entre trabajo y hogar, seobtiene la siguiente situación (UNESCO 2008).

Estos resultados evidencian la extensa jornada laboral del docente y el tiempo libre disponible para los profesores para el hogar que es muy escaso.

Las condiciones de trabajo de cualquier empleado público o privado son primordiales, y con más razón del docente que constantemente se halla trabajando con personas, ya sean, padres, alumnos, colegas. Por ello, el trabajo de investigación, se estarán abordando las condiciones de trabajo como el amplio escenario de convergencia entre la salud, el tiempo laboral, a la luz de las estadísticas presentadas por otros países latinoamericanos, tales como Chile, Ecuador, Argentina, entre otros.

Así se plantea que las condiciones de trabajo es un escenario donde convergen un conjunto de dimensiones sociales, personales y físicas en las cuales laboran los docentes y la salud como un concepto integral que depende de un equilibrio social, psicológico, fisiológico y biológico que influye, fuertemente, en la manera como los docentes acuden a trabajar. Los pocos estudios latinoamericanos disponibles sobre el tema, entre otros los realizados en Argentina, Chile, Ecuador, México, representan una voz de alarma para el sistema educativo y la sociedad en su conjunto debido a que ofrecen hallazgos múltiples, en particular relacionados con la afección de la salud mental expresada en enfermedades como estrés, depresión, neurosis y una variedad de enfermedades psicosomáticas diagnosticadas y percibidas (gastritis, úlceras, colon irritable, entre otras) (UNESCO 2010).

\subsubsection{Condiciones materiales}

La seguridad y salud en el trabajo es un área interdisciplinaria relacionada con la seguridad, la salud y la calidad de vida en el empleo, al respecto:

Un rubro a considerar tiene que ver con las condiciones materiales, en especial lo que se refiere al estado de la infraestructura y al mantenimiento de las aulas, patios y sanitarios, que conjuntamente con el número de alumnos por clase y los volúmenes de trabajo de los profesores, según la opinión internacional, pueden representar condiciones poco favorables para la enseñanza y el aprendizaje. En torno al mismo asunto, desde hace 
varias décadas la Recomendación OITUNESCO, relativa a la situación del personal docente, ha insistido en que "los edificios escolares deberían reunir las garantías de seguridad necesarias, resultara gradables... y ser de uso funcional... estar construidos con materiales duraderos y según las normas higiénicas"

Lo anterior no se cumple, en tanto se observan escuelas con aulas en peligro de derrumbe y planteles que carecen de sanitarios exclusivos para los maestros. La dotación de recursos pedagógicos es otro rubro a considerar. En las escuelas guanajuatenses, la mayor parte del material que se utiliza en el trabajo pedagógico provisto por los mismos profesores. Además, la mayoría de los docentes (72\%), comentan que dicho recurso es insuficiente (UNESCO 2010).

Uno de los factores menos estudiados, pero de gran relevancia por las implicaciones en el profesorado, se refieren a las exigencias inherentes al trabajo en el aula. Desde la perspectiva de los profesores, las actividades que realizan los expone de manera cotidiana a forzar la voz (99\%), estar de pie durante toda la jornada (96\%), trabajar en ambientes ruidosos (72\%), con temperatura inadecuada (79\%), y en ocasiones también con iluminación deficiente (58\%) (Diálogos Educativos, 2012).

De acuerdo la Constitución Nacional del Paraguay, la Salud del trabajador es un derecho de toda persona.

\subsubsection{Condiciones fisiológicas}

Existe una amplia literatura que demuestra, por una parte, la influencia de las condiciones de trabajo y la salud en el rendimiento laboral. En el campo de la educación estos estudios son recientes y escasos, entre otras razones porque históricamente la docencia se ha configurado como un apostolado, como un "servicio social" más que como un trabajo para el cual se requería de calificaciones, estándares de desempeño y procesos de evaluación. El concepto de profesionalidad del trabajo docente surge, relativamente, hace poco tiempo, en medio de los debates acerca de la calidad de la educación y su relación con el desarrollo. Esta interpretación de la docencia como apostolado lleva, implícitamente, un sentido intrínseco de sacrificio y renuncia. Trabajar en condiciones inadecuadas, recorrer enormes distancias hasta su escuela, contar con recursos didácticos rudimentarios, padecer enfermedades derivadas del ejercicio, etc. era parte de lo que estaba (o aún está) dispuesto a aceptar aquel o aquella que decidía optar por la docencia. Disfonía, várices, dolores lumbares, fatiga, han sido y son 
asumidas como las inevitables "marcas" de la profesión contra las cuales no hay nada que hacer. Pero, simultáneamente, ha significado que durante largo tiempo se considere que para ejercer la docencia la vocación bastaba; que era una profesión que no demandaba conocimientos demasiado complejos ni aprendizaje permanente, tampoco uso de recursos tecnológicos y que, además, era un trabajo de "medio tiempo", lo cual explica en mucho la composición de género del profesorado (Diálogos Educativos, 2012).

Las condiciones de trabajo de los docentes son consideradas una problemática social, al escuchar el discurso de los propios profesores, puesto que es de mucha importancia como ellos viven el día a día en sus escuelas. Por esta razón la incorporación de los profesores resulta clave en esta investigación, siendo el centro en el proceso del trabajo docente. Las principales transformaciones que ha experimentado la profesión docente en los últimos tiempos, permiten efectuar una discusión sobre el papel del docente en su trabajo diario.

Tiempo disponible para desarrollar actividades académicas

La labor docente requiere de gran responsabilidad y de toda una planificación para desarrollar las actividades académicas, al respeto, para los países latinoamericanos, plantea la UNESCO, lo siguiente:

El Proyecto Regional de Educación para América Latina y el Caribe (PRELAC), la carta de navegación para los sistemas educativos, aprobada y firmada por los ministros de educación de la región en el 2002, destaca el papel de los docentes al definir como segundo foco estratégico "el fortalecimiento del protagonismo docente para que respondan a las necesidades de aprendizaje de los estudiantes" (UNESCO, 2010).

\section{Vocación docente y apoyo de la familia en el logro educativo de los alumnos}

Entre estos nuevos desafíos, se puede afirmar que, sólo como consecuencia de la pérdida de ciertas funciones (económicas y políticas, entre otras), la familia ha podido encontrar su función o funciones propias y que justamente por ello ha también aumentado su importancia social (Tyrell, 2017).

Según Pizarro; Santana y Vial (2013) para dar cuenta de la relevancia de la familia en los procesos de aprendizaje es necesario detenerse en la relación familia-escuela esta vinculación ha sido cambiando conforme ha cambiado la sociedad en su conjunto: 
"Las primeras escuelas mantenían una estrecha unión con la comunidad, a partir del siglo XX comenzaron a distanciarse. La labor pedagógica se fue especializando y haciendo cada vez más compleja, y los maestros enseñaban materias y utilizaban métodos alejados de la experiencia de los padres y madres, que poco tenían que decir acerca de lo que ocurría en las aulas” (Maestre, 2009, p. 6).

Este alejamiento se mantuvo, incluso argumentando que familia y escuela buscaban objetivos divergentes. Hoy se plantea que tienen influencias superpuestas responsabilidades compartidas y por tanto se debe definir la relación bajo condiciones de colaboración (Pizarro; Santana y Vial 2013).

De acuerdo a Ospina Botero y Montoya Pavas (2017) explica que:

De esta manera, pensar en las prácticas educativas es pensar en múltiples dimensiones: los actores primarios del hecho educativo (los docentes facilitadores, padres-madres de familia, actores comunitarios, otros), es pensar en los contenidos a comunicar o compartir, en los objetivos que se propone la acción educativa, en las formas organizativas que adquiere la tarea de enseñar y aprender, en los contextos donde se desarrolla; es pensar también en los recursos utilizados para comunicar el conocimiento. Pero fundamentalmente, es considerar la dimensión significativa de esta práctica, es decir, en la potencialidad de una construcción intersubjetiva de sentidos que para el caso particular de la investigación de donde surge este artículo, está vinculada con los propósitos que definen las prácticas educativas en la promoción del desarrollo familiar. Esta consideración por las prácticas educativas implementadas con las familias pone al descubierto experiencias exitosas y con un valioso impacto que, en una tarea sencilla y comprometida por parte de las instituciones, no ha sido suficientemente valorada y dimensionada en sus riquezas pedagógicas y que en 
últimas se pueden convertir en puntos de referencia para las instituciones pertinentes en el tema (p.9).

De esta forma, se comprende que la familia es aquel escenario que introduce a los miembros más pequeños en el mundo de la socialización, por lo que para lograr que los individuos interioricen normas, pautas de convivencia y se comuniquen de manera asertiva, es importante primero que estas se vivan y sean fomentadas al interior de la familia, por lo que es importante que las familias compartan diferentes espacios de interacción donde se repliquen estos elementos (Ospina Botero y Montoya Pavas 2017).

\section{METODOLOGÍA}

La investigación se enmarca en un estudio de tipo descriptivo correlacional, con la finalidad de describir características propias de las variables en estudio.

Según Arias (2004) el estudio descriptivo identifica características del universo de investigación, señala formas de conducta y actitudes del universo investigado, descubre y comprueba la asociación entre variables de investigación (p.133).

Mendoza, Machado, Montes, (2016), abordan el tema de las necesidades de un plan de vida para la orientación profesional, para ello hacen una revisión de varias literaturas y logran caracterizar el plan de vida y desarrollar la hipótesis.

La presente investigación adopta un diseño no experimental, siendo que se observó situaciones existentes, no manipuladas por el investigador, tal y como se da en su contexto natural para luego analizarlas (Sampieri y otro, 2003).

Este diseño permite visualizar los obstáculos evidenciados entre los docente, alumnos y padres para permitir un aprendizaje efectivo de los alumnos y los medios que posee y busca el docente para logar el aprendizaje efectivo de los alumnos.

En cuanto a la cronología en el tiempo, esta investigación es de corte trasversal o transaccional, ya que se estudiará en un periodo de 2020-2021

Al respecto Sampieri y otros (2014) señala que este diseño tiene como objetivo indagar la incidencia y los valores en que se manifiesta una o más variables en un momento determinado.

La presente investigación abordará una metodología de enfoque mixto; el cualitativo y el cuantitativo, de manera a describir, desde diferentes perspectivas la problemática enunciada. 
Esta investigación es cualitativa, así como (Gómez, Flores, Giménez, 1996) y (Hernández, 2005) detallan que la metodología de la investigación cualitativa, en resultados, se analizan de manera cualitativa, categorizándose las respuestas en función de los indicadores de las dimensiones estudiadas. Así los datos cuantitativos representan los datos numéricos y estadísticos que requiere la investigación.

Población y Muestra: Conforman 231 alumnos de la Educación Escolar Básica, 22 docentes, 1 evaluadora 1 director, y 60 padres de la Escuela Básica No 578 "Defensores del Chaco", San Lorenzo - Pilar.

Para la selección de la muestra se optó por un muestreo aleatorio siempre tomando el $30 \%$ de la población estudiantil, la que corresponde a 60 padres, 69 alumnos/as y el muestreo no probabilístico intencional, en el caso de docentes (22), evaluadora (1), director (1).

Según (Cuesta, 2009) El muestreo no probabilístico es una técnica de muestreo donde las muestras se recogen en un proceso que no brinda a todos los individuos de la población iguales oportunidades de ser seleccionados.

\section{RESULTADOS Y DISCUSIÓN}

\section{Capítulo IV- Análisis y presentación de resultados}

Luego del análisis de los instrumentos de recolección de datos aplicado a los diferentes estamentos de la institución en estudio, a continuación, se presenta los resultados de la investigación conforme a las variables estudiadas:

Los resultados de las puntuaciones resultan de la siguiente sumatoria: Puntuación General: 17 preguntas por 153 sujetos encuestados y 3 niveles de opción respuesta. Totalizando así: 7.803 puntos posibles, si es que todos optan por el nivel máximo 3, en su respuesta.

Los análisis se efectúan primeramente por dimensión en tablas y luego en gráficos de manera a establecer e interpretar sus principales relaciones. En ese sentido, se ha aplicado el análisis estadístico en su perspectiva descriptiva, como instrumento técnico especializado para caracterizar las dimensiones de cada variable. Por último, se realiza un análisis general, con miras a una armonización global de los resultados de las dimensiones investigadas de la variable principal.

Las puntuaciones se agrupan por dimensión, subdimensión e indicador, según la opción, dónde: 1: Nunca 2: A veces; 3: Siempre. 
- Respecto a la variable: Influencia de la vocación docente en el aprendizaje de los alumnos.

Figura 1. Distribución de los puntajes en porcentajes, relacionados a la Dimensión1: "Influencia de la vocación docente en el aprendizaje de los alumnos", agrupadas por subdimensiones según opciones de respuestas.

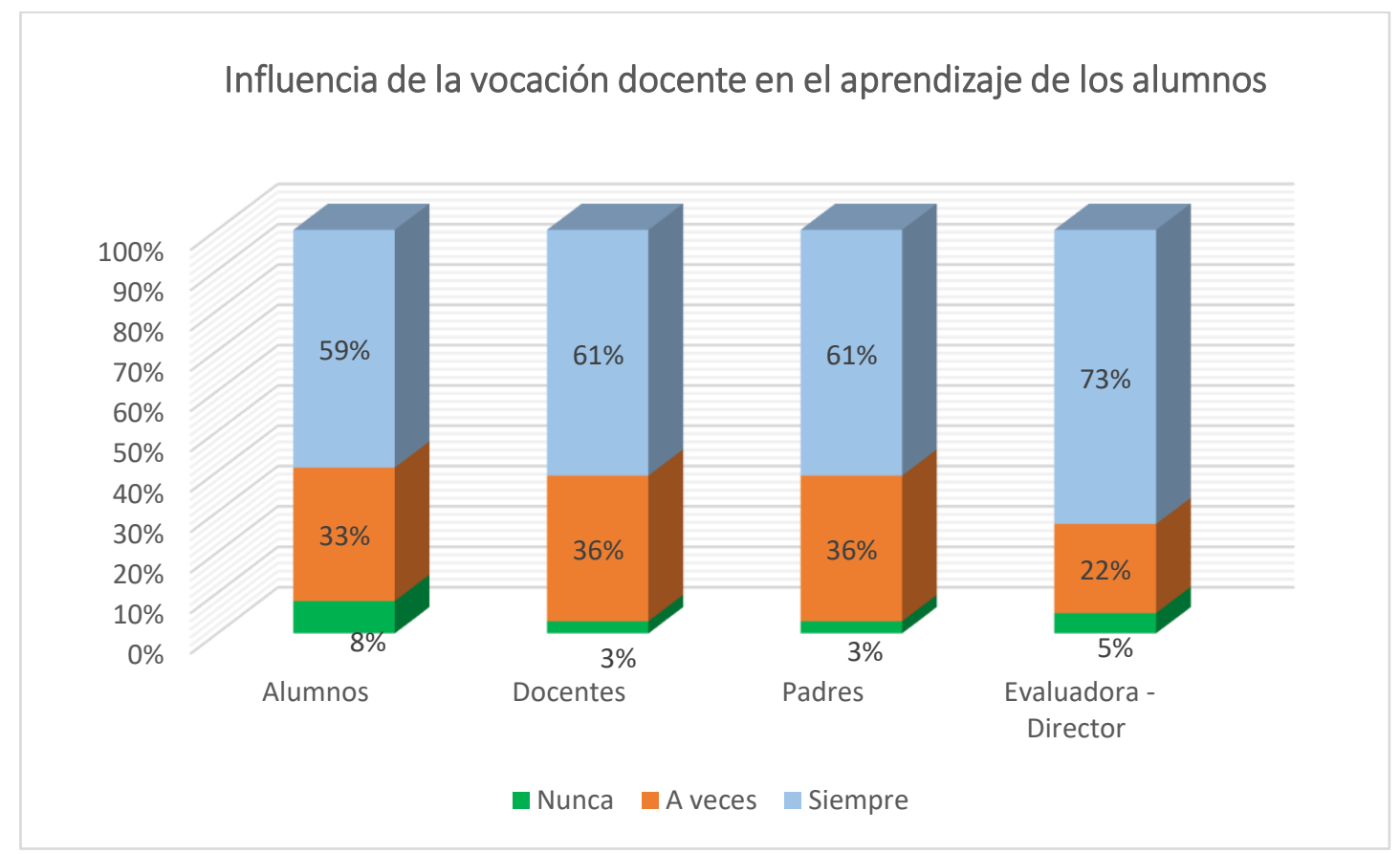

Al efectuar un análisis enfocado a la subdimensión: proceso de articulación y sus indicadores: Vocación, Aptitudes, Voluntad, Conocimiento, Desempeño y Rendimiento en el trabajo; según la escala de valoración, se observa un rango de afirmativo de tres niveles en primer lugar la opción siempre el porcentaje más relevante que corresponde a la estructura de cada nivel y los demás indicadores en porcentajes positivos interiores, evidenciándose un nivel alto de la escasa respecto a la variable influencia de la vocación docente en el aprendizaje de los alumnos.

En concordancia con lo expuesto (Álvarez, 1995), al referirse a la Orientación Vocacional también como un proceso de desarrollo de la carrera a lo largo de la vida de las personas afirma a que está presente en los diferentes contextos en que se encuentren éstas, a saber

- El Educativo: que abarca el período formativo, es decir la preparación para la vida laboral y que se desarrolla fundamentalmente en el contexto escolar y comunal. 
- Dentro de este contexto la Orientación Vocacional desarrolla diferentes acciones con el estudiantado, padres, madres, personas encargadas y docentes con el fin de favorecer el desarrollo integral del estudiantado y dentro de éste el vocacional.

El Organizacional: incluye el período de desempeño laboral que tiene lugar en el centro de trabajo y en el cual se dan gran parte de las experiencias de las personas relacionadas precisamente con el trabajo, el cual cumple una doble función, tanto a nivel individual como social, de ahí su importancia.

- Respecto a la variable: Acompañamiento de padres o tutores con los docentes para el logro del aprendizaje

Figura 2. Distribución de los puntajes en porcentajes, relacionados a la Dimensión 2: "Acompañamiento de padres o tutores con los docentes para el logro del aprendizaje", agrupadas por subdimensiones según opciones de respuestas.

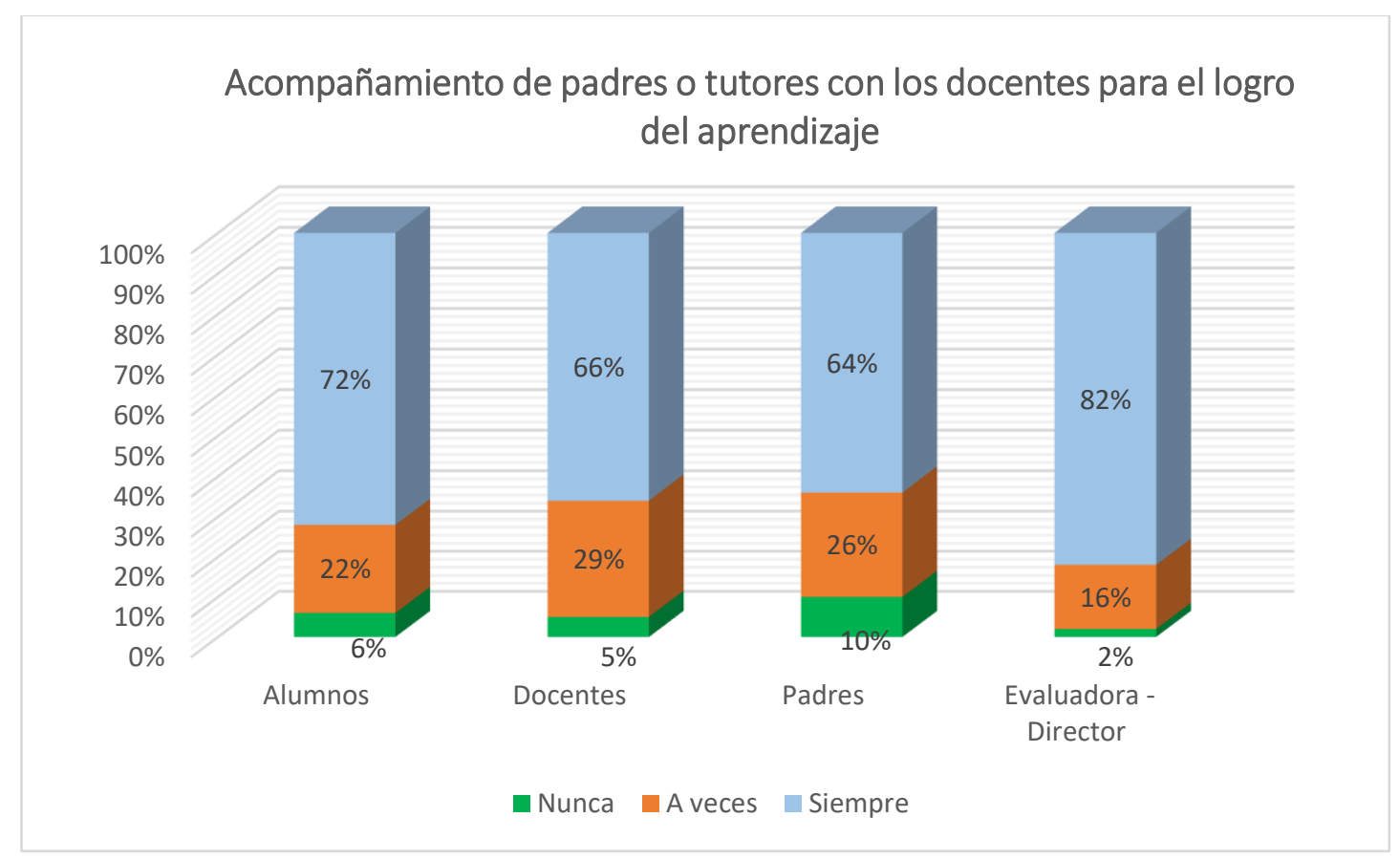

Al efectuar un análisis enfocado a la variable: "Acompañamiento de padres o tutores con los docentes para el logro del aprendizaje" y sus indicadores: Actitud de confianza entre docentes-padres -alumnos, relación comunicativa docente-alumno, relación comunicativa padres de familia -profesores/as, estrategias de enseñanza, necesidades del profesorado, tales como necesidades tecnológicas y conectividad de los alumnos, seguido del indicador apoyo de padres y experiencias familiares, según la escala de valoración, se observa un rango de afirmativo superior al $60 \%$, lo que contrastando con la teoría, entre otros autores, se puede afirmar que, sólo como consecuencia de la 
pérdida de ciertas funciones (económicas y políticas, entre otras), la familia ha podido encontrar su función o funciones propias y que justamente por ello ha también aumentado su importancia social (Tyrell, 2017).

De esta manera, pensar en las prácticas educativas es pensar en múltiples dimensiones: los actores primarios del hecho educativo (los docentes facilitadores, padres-madres de familia, actores comunitarios, otros), es pensar en los contenidos a comunicar o compartir, en los objetivos que se propone la acción educativa, en las formas organizativas que adquiere la tarea de enseñar y aprender, en los contextos donde se desarrolla; es pensar también en los recursos utilizados para comunicar el conocimiento. Pero fundamentalmente, es considerar la dimensión significativa de esta práctica, es decir, en la potencialidad de una construcción intersubjetiva de sentidos que para el caso particular de la investigación de donde surge este artículo, está vinculada con los propósitos que definen las prácticas educativas en la promoción del desarrollo familiar (Ospina Botero y Montoya Pavas, 2017).

Esta consideración, expuesta por el mismo autor, por las prácticas educativas implementadas con las familias pone al descubierto experiencias exitosas y con un valioso impacto que, en una tarea sencilla y comprometida por parte de las instituciones, no ha sido suficientemente valorada y dimensionada en sus riquezas pedagógicas y que en últimas se pueden convertir en puntos de referencia para las instituciones pertinentes en el tema.

De esta forma, se comprende que la familia es aquel escenario que introduce a los miembros más pequeños en el mundo de la socialización, por lo que para lograr que los individuos interioricen normas, pautas de convivencia y se comuniquen de manera asertiva, es importante primero que estas se vivan y sean fomentadas al interior de la familia, por lo que es importante que las familias compartan diferentes espacios de interacción donde se repliquen estos elementos (Ospina Botero y Montoya Pavas 2017).

- Respecto a la variable: Sentido de la vocación y la profesión del docente en el marco de la retención del alumno. 
Figura 3. Distribución de los puntajes en porcentajes, relacionados a la Dimensión 3: "Sentido de la vocación y la profesión del docente en el marco de la retención del alumno", agrupadas por subdimensiones según opciones de respuestas

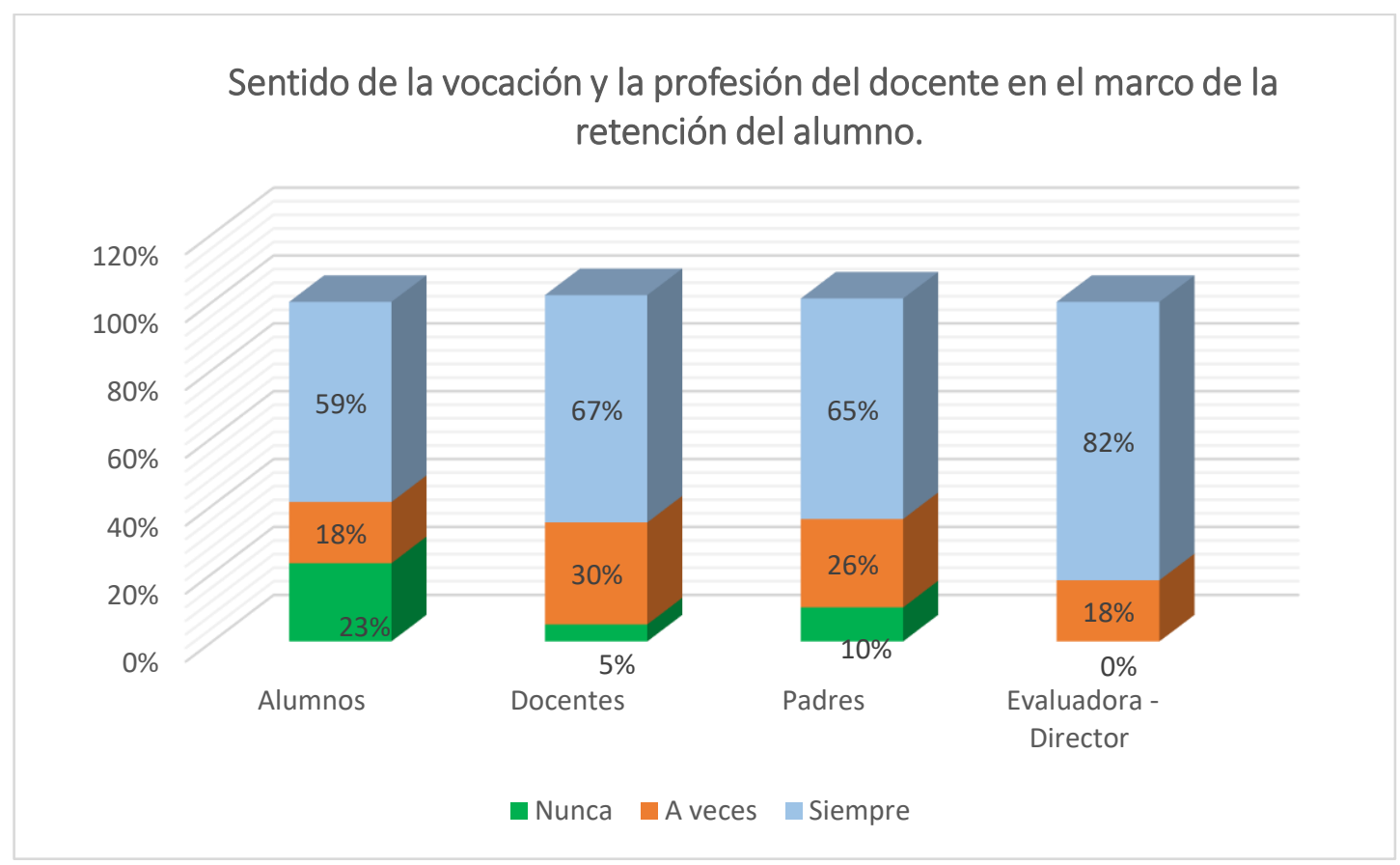

Al efectuar un análisis enfocado a la subdimensión Sentido de la vocación y la profesión del docente en el marco de la retención del alumno”, con sus indicadores: Estrategias de enseñanza, fracaso escolar, motivación, intervención de organismo del estado, éxito escolar y condición económica no se ha alcanzado un nivel adecuado del $60 \%$ del estamento estudiantil

de los indicadores mencionados lo que evidencia la existencia de la necesidad de mayor sentido de la vocación y la profesión del docente en el marco de la retención del alumno de la institución en estudio.

Es indiscutible que la calidad educativa, y particularmente los resultados educativos de los estudiantes, son aspectos complejos donde intervienen variedad de factores provenientes del propio estudiante, del profesor, del currículo y de las políticas educativas, entre otros (Martínez, 2004; Valdés, Urías, Carlos y Tapia, 2009, mencionado por (Valdés Cuervo y Urías Murrieta, 2011); así mismo, se reconoce la influencia de los factores contextuales de los estudiantes, en especial los referidos al nivel socioeconómico y el capital cultural de la familia. 
En las últimas décadas, la investigación educativa ha aportado un número creciente de evidencias sobre la importancia que la cooperación entre familia y centro escolar tiene para el logro de buenos resultados educativos. En los años 80, los estudios enmarcados en el movimiento de eficacia escolar otorgaron protagonismo a esta cuestión, al determinar que uno de los rasgos que caracteriza a las escuelas de éxito es que cuentan con familias que muestran una fuerte implicación en la vida escolar de sus hijos e hijas y que tienden a participar activamente en los centros educativos (Mortimore et al., 1988, mencionado por Gálvez y Bertran, 2017).

\section{CONSIDERACIONES FINALES}

Por tanto, respecto a la influencia de la vocación docente en el aprendizaje de los alumnos, en lo que se ha determinado establecer los indicadores de vocación, aptitudes, voluntad, conocimiento y desempeño del docente, rendimiento en el trabajo, se ha observo un nivel recurrente de la opción siempre con 59\%, 61\%, en igual porcentaje del estamento docente y padres el $61 \%$ y el $73 \%$ de evaluador y director, los demás indicadores en porcentajes positivos interiores a estos porcentajes, evidenciándose un nivel alto respecto a la variable influencia de la vocación docente en el aprendizaje de los alumnos, indicando la gran influencia la vocación del docente, que a través de su desempeño laboral en el trabajo puede incluir en los estudiantes, permitiendo su retención, cumpliendo su labor social y permitir la graduación del escolar.

A partir de ese momento, muchos trabajos han señalado que la colaboración tiene efectos beneficiosos no solo para los estudiantes, sino también para las familias y para las propias escuelas, como muestran diversas revisiones de investigación realizadas al respecto (Halgunseth et al., 2009, mencionado por Gálvez y Bertran, 2017).

En cuanto al acompañamiento de padres o tutores con los docentes para el logro del aprendizaje, con sus indicadores: actitud de confianza entre docentes-padres -alumnos, relación comunicativa docente-alumno, relación comunicativa padres de familia profesores/as, estrategias de enseñanza, necesidades del profesorado, tales como necesidades tecnológicas y conectividad de los alumnos, seguido del indicador apoyo de padres y experiencias familiares, según la escala de valoración, se observa que el Nivel 3 de la Escala: (Siempre) es la opción más recurrente de los encuestados; ubicándose así en primer lugar, lo que existe un alto nivel de estrategias de enseñanza implementada por los docentes para evitar el fracaso escolar, así también se ha 
observado la intención de padres, a través de la motivación a sus hijos para proseguir con sus estudios, en ningún caso se ha observado o no ha existido necesidades de la intervención de organismos del estado como la o Fiscalía para recurar al alumnos, siendo que hasta la fecha no existe abondo escolar, en cuanto a la situación socioeconómica de los mismos es mantiene en un rango medio.

De esta manera se ajusta a lo expuesto por los autores como (Ospina Botero y Montoya Pavas, 2017), donde exponen que pensar en las prácticas educativas es pensar en múltiples dimensiones: los actores primarios del hecho educativo (los docentes facilitadores, padres-madres de familia, actores comunitarios, otros), es pensar en los contenidos a comunicar o compartir, en los objetivos que se propone la acción educativa, en las formas organizativas que adquiere la tarea de enseñar y aprender, en los contextos donde se desarrolla; es pensar también en los recursos utilizados para comunicar el conocimiento. Pero fundamentalmente, es considerar la dimensión significativa de esta práctica, es decir, en la potencialidad de una construcción intersubjetiva de sentidos que para el caso particular de la investigación de donde surge este artículo, está vinculada con los propósitos que definen las prácticas educativas en la promoción del desarrollo familiar.

Respecto al sentido de la vocación y la profesión del docente en el marco de la retención del alumno, estudiando los indicadores como estrategias de enseñanza, fracaso escolar, motivación, intervención de organismo del estado, éxito escolar y condición económica, según la escala de valoración, se ha observado que el nivel 3 de la Escala: (Siempre) es la opción más recurrente de los encuestados; ubicándose así en primer lugar. Sin embargo, no se ha alcanzado un nivel adecuado del $60 \%$ del estamento estudiantil de los indicadores mencionados lo que evidencia la existencia de la necesidad de mayor sentido de la vocación y la profesión del docente en el marco de la retención del alumno de la institución en estudio.

De esta manera se ha llegado a la conclusión confirmando la hipótesis que plantea: La vocación docente, el acompañamiento coordinado de padres o tutores con los docentes y organismos del estado influyen de manera positiva en el aprendizaje de niños/as de la Escuela Básica No 578 “Defensores del Chaco”, San Lorenzo Pilar, año 2020-2021, en tiempo de pandemia. 
Así como señala (Marqués, 2004), explicando que el docente es una persona que recurre en sus funciones con buenas prácticas que le permiten lograr buenos resultados, proyectados a una formación de calidad; es una persona activa, con habilidades sociales que promueve la participación de los estudiantes en procesos de enseñanza aprendizaje; es capaz de transmitir una disciplina de superación ante las dificultades, y es persistentes en el logro de soluciones; es creativo, reflexivo, crítico y promueve este pensamiento; es capaz de crear situaciones y contextos que se aproximen a la realidad que viven los estudiantes y también a la de los contextos laborales; utiliza una gran variedad de estrategias didácticas, implicando diversidad de recursos que faciliten los procesos de enseñanza-aprendizaje, por lo tanto, debe ser un profesional capaz de reflexionar críticamente su propia práctica pedagógica, en busca de guiar a sus estudiantes al logro de competencias necesarias para la inserción en la sociedad en permanente evolución, que demanda competencias docentes que se configuran desde lo humano y lo profesional.

\section{REFERENCIAS BIBLIOGRÁFICAS}

Acosta, Savier F.; García, M Ch, (2012), Estrategias de enseñanza utilizadas por los docentes de biología en las universidades públicas Omnia, vol. 18, núm. 2, mayo-agosto, 2012, pp. 67-82 Universidad del Zulia Maracaibo, Venezuela

Aguilera, A. (2004). Introducción a las Dificultades del Aprendizaje, GrawHill/Interamericana. Madrid.

Alcántara (2015). Experiencias adquiridas sobre formación vocacional y orientación profesional de las ciencias médicas en Villa Clara. Edumecentro 2015;7(2):132147 ISSN 2077-2874 RNPS 2234.

Álvarez,. (1995). Orientación Profesional. España: Editorial Cedecs.

Arias, F. (2006). El Proyecto de Investigación $6^{\mathrm{a}}$ Edición. Editorial: Episteme. Venezuela

Ávila, D. (2016). Familia y grupos de pares: otros protagonistas en la orientación profesional de los estudiantes de la Enseñanza Media Superior cubana. Revista Iberoamericana de Educación / Revista Ibero-americana de Educação vol. 71, núm. 1 (15/05/16), pp. 133-156, ISSN: 1022-6508 / ISSNe: 1681-5653. Organización de Estados Iberoamericanos. 
Bazán, A, Backhoff, E, \& Turullols, R (2016). Participación escolar, apoyo familiar y desempeño en Matemáticas: El caso de México en PISA (2012). RELIEVE. Revista Electrónica de Investigación y Evaluación Educativa, 22(1),1-17.[fecha de Consulta 17 de Febrero de 2020]. ISSN: . Disponible en: https://www.redalyc.org/articulo.oa?id=916/91649056007

Cano González, Rufino; Casado González, Mónica. (2015). Escuela y familia. Dos pilares fundamentales para unas buenas prácticas de orientación educativa a través de las escuelas de padres Revista Electrónica Interuniversitaria de Formación del Profesorado, vol. 18, núm. 2, mayo-agosto, 2015, pp. 15-28 Asociación Universitaria de Formación del Profesorado Zaragoza, España

Carrillo, M; Padilla, J; Rosero, T; Villagómez, M, (2009). La motivación y el aprendizaje ALTERIDAD. Revista de Educación, vol. 4, núm. 2, juliodiciembre, 2009, pp. 20-32 Universidad Politécnica Salesiana Cuenca, Ecuador

Chong González, E. G. (2017). Factores que inciden en el rendimiento académico de los estudiantes de la Universidad Politécnica del Valle de Toluca. Revista Latinoamericana de Estudios Educativos (México), vol. XLVII, núm. 1, 2017, pp. 91-108 Centro de Estudios Educativos, A.C. Distrito Federal, México

Diálogos Educativos, $\quad \mathrm{N}^{\circ} \quad 24, \quad$ año $2016 \quad$ (en línea) http://www.dialogoseducativos.cl/revistas/n24/rodriguez).

Espitia Carrascal, R. E.; Montes Rotela, M. (2009). Influencia de la Familia en el Proceso Educativo de los Menores del Barrio Costa Azul de Sincelejo (Colombia) Investigación \& Desarrollo, vol. 17, núm. 1, 2009, pp. 84-105 Universidad del Norte Barranquilla, Colombia.

Fernández, Y., González, N., Pompa V., Garcés, V., Figueredo, E., (2016). Motivación profesional en estudiantes de enfermería. Revista Multimed Médica Granma. ISSN 1028-4818 RPNS-1853.

Gálvez, E, Bertran Tarrés, I. M, (2017). Prácticas de colaboración familia-escuela en centros de éxito de entornos desfavorecidos pedagogía social. Revista interuniversitaria, núm. 29, enero-junio, 2017, pp. 97-110 Sociedad Iberoamericana de Pedagogía Social Sevilla, España

Gómez, G., Flores, J, Giménez, E. (1996). Metodología de la Investigación Cualitativa. Barcelona: Tauro. 
González R., Cardentey, J. (2015). La orientación vocacional en residentes de Medicina General Integral. Revevista Arch Med Camagüey Vol19(6)2015.

Hernández Arteaga, I; Recalde Meneses, J; Luna, J A. (2015). Estrategia Didáctica: Una Competencia Docente en la Formación para el Mundo Laboral Revista Latinoamericana de Estudios Educativos (Colombia), vol. 11, núm. 1, enerojunio, 2015, pp. 73-94 Universidad de Caldas Manizales, Colombia

Hernández Sampieri, R. (2005 ).Metodología de la Investigación. La Habana: Félix Varela.

Larrosa Martínez, F. (2010). Vocación docente versus profesión docente en las organizaciones educativas. Revista Electrónica Interuniversitaria de Formación del Profesorado, vol. 13, núm. 4, 2010, pp. 43-51 Asociación Universitaria de Formación del Profesorado Zaragoza, España

Martín F., M. M., Dapelo B., Pizarro J., Gusto C., \& otros (2015). El constructo personalidad eficaz: últimos avances. Revista Orientación Vocacional 29: 52-68.

Medina, C., Watanabe, R., Angulo C., (2018). Influencia de la vocación profesional en el rendimiento académico de los estudiantes de Medicina Veterinaria y Zootecnia de una universidad privada de Lima, Perú. Revista Inv Vet Perú 2018; 29(4): 1073-1086. Disponible en: http://dx.doi.org/10.15381/rivep.v29i4.15193.

Mendoza, I. Machado, E. Montes, N. (2016). La orientación vocacional y la elaboración de los proyectos personales de vida. Tendencias y enfoques la orientación vocacional y la elaboración de los proyectos personales de vida. Revista Cognosis; Filosofía, Letras y Ciencias de la Educación.

Ospina Botero, M y Montoya Pavas, E, (2017). Las prácticas educativas con familia desde la escuela. Universidad Católica de Pereira, Colombia. Instituto de Estudios en Educación Universidad del Norte

Pérez I., Rodríguez F. (2016). La identidad profesional como configuración subjetiva de la personalidad de los estudiantes de la carrera de enfermería. Cuadernos de Educación y Desarrollo 19(2). Disponible en: http://www.eumed.net/rev/ced/19/prrb2.htm.

Pizarro Laborda, P; Santana López, A; Vial Lavín, B. (2013). La participación de la familia y su vinculación en los procesos de aprendizaje de los niños y niñas en 
contextos escolares Diversitas: Perspectivas en Psicología, vol. 9, núm. 2, 2013, pp. 271-287 Universidad Santo Tomás Bogotá, Colombia

Ramírez, María Aurelia (2005). Padres y Desarrollo de los Hijos: Practicas de Crianza Estudios Pedagógicos, vol. XXXI, núm. 2, 2005, pp. 167-177 Universidad Austral de Chile Valdivia, Chile.

Real Academia Española. (2014). Diccionario de la Real Academia Española (23 ed.). Madrid.

Rodríguez M., Trujillo P, Bolaños P., Leyva M. (2015). La orientación profesional: indicador de eficiencia en la formación de profesionales. Revista Edumecentro 7(4):178-95.

Disponible

en: http://www.revedumecentro.sld.cu/index.php/edumc/article/view/449/pdf_1

Rodríguez, M, (2010). Factores personales y familiares asociados a los problemas de comportamiento en niños. Universidad de la Sabana, Facultad de Psicología, Centro de Servicios de Psicología. Campus Universitario del Puente del Común, Colombia.

Rodríguez, M. R. (2013). Influencia de la implicación de la familia en la motivación de los hijos. Trabajo de Investigación de la Facultad de Ciencias de la Educación, Enfermería y Fisioterapia Universidad de Almería. Maestría de Intervención en Convivencia Escolar.

Ruíz De Miguel, Covadonga, (2001). Departamento de Métodos de Investigación y Diagnóstico en Educación Facultad de Educación Universidad Complutense. Revista Complutense de Educación ISSN: 1130-2496 Vol, 12 Núm. 1(2001): 81-113 Factores familiares vinculados al bajo rendimiento.

Tyrell, H; V. (2017). La Familia y escuela: Algunas reflexiones sobre la diferenciación interna del sistema de la educación Revista Mad. Revista del Magíster en Análisis Sistémico Aplicado a la Sociedad, núm. 36, 2017, pp. 1-20 Facultad de Ciencias Sociales Santiago de Chile, Chile

UNESCO, (2008). Estudios de Casos en Argentina, Chile, Ecuador, México, Perú y Uruguay

Valbuena Cueto, Vidaura, (2008). Desarrollo de actividades en el aula y el proceso de construcción del conocimiento en alumnos de Educación Básica Omnia, vol. 14, núm. 3, 2008, pp. 9-31 Universidad del Zulia Maracaibo, Venezuela. 
Valdivieso-León, L; R, Flores L, Valle; Aken, M. Van A. G. (2016). Prácticas educativas familiares: ¿cómo las perciben los padres? ¿cómo las perciben los hijos? ¿qué grado de acuerdo hay? Perspectiva Educacional, Formación de Profesores, vol. 55, núm. 1, enero, 2016, pp. 129- 151 Pontificia Universidad Católica de Valparaíso Viña del Mar, Chile

Vargas, M.M. \& Montero, E. (2016). Factores que determinan el rendimiento académico en Matemáticas en el contexto de una universidad tecnológica: aplicación de un Modelo de Ecuaciones Estructurales. Universitas Psychologica , 15 (4). http://dx.doi.org/10.111 44/Javeriana.upsy15-4.fdra

Velásquez, T., Puentes, A., Sarabia, J. (2014). Orientación vocacional aplicando sistemas basados en conocimiento. Revista Colombiana de Tecnologías de Avanzada, ISSN: 1692-7257 - Volumen 1 - Número 23 - 2014. 